\title{
Fatigue reliability assessment of small sample excavator working devices based on Bootstrap method
}

\author{
Yuhong Shao, Pengmin Lu, Binhua Wang, Qingyi Xiang \\ Key Laboratory of Road Construction Technology and Equipment, Ministry of Education, Chang'an University, Xi'an 710064, \\ China \\ Shaoyb@chd.edu.cn,_pmin@chd.edu.cn,wangbb@chd.edu.cn,383393093@qq.com
}

\begin{abstract}
To evaluate the fatigue reliability of the excavator working device, the fatigue tests of 2 sets of moving arm and bucket rod of medium-sized excavators with self-weight of 26000 kgwere carried out. The virtual augmented sample method (VASM) combined with Bootstrap method was used to analyze the reliability of the excavator working device under extreme small samples, and the interval and point estimations of life parameters were obtained. Based on the lognormal distribution of the excavator working device, the reliability evaluation model of the excavator working device was established, and reliability indexes, such as reliability function, failure distribution function, inefficiency function, reliable life and so on, were obtained. And the results of fatigue safety life under different confidence and reliability were calculated. The evaluation results show that the average failure time of the excavator working device is 6124 hours under the confidence of $75 \%$, which provides an important reference for the design, the safety inspection and maintenance decision of the excavator working device.
\end{abstract}

KEYWORDS. Fatigue reliability; Small sample; Bootstrap; Excavator working device; F test.

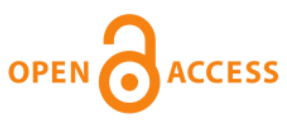

Citation: Shao Y.H., Lu P.M., Wang B.H., Xiang Q.Y., Fatigue reliability assessment of small sample excavator working devices based on Bootstrap method, Frattura ed Integrità Strutturale, 48 (2019) 757-767.

Received: 24.01.2019

Accepted: 21.03 .2019

Published: 01.04.2019

Copyright: (C) 2019 This is an open access article under the terms of the CC-BY 4.0, which permits unrestricted use, distribution, and reproduction in any medium, provided the original author and source are credited.

\section{INTRODUCTION}

he working device of the excavator is the direct actuator of the excavator operation, and the reliability of the working device is one of the key indexes of the excavator's quality. Therefore, it is of great significance to study the reliability of the excavator's working device [1]. Due to the high cost of the excavator working device and the limitation of the test cost and time, the fatigue life test can only be carried out by using $2 \sim 3$ test pieces rather than the large sample size test data to meet the statistical requirements. As a result, the fatigue reliability evaluation of excavator working device is a problem of very small sample. 
The existing reliability assessment methods include Semi-empirical method [2], Bayes method [3], student-t distribution method [4], Bootstrap method [5] and so on. Under the condition of no empirical data, the student-t distribution method can be used in the case of the small sample with the unknown parent variance to deduce the interval estimation of the overall mean of the set confidence level. However, the confidence interval estimated by this method is often wider. The Bootstrap method is a statistical method that only relies on sample information proposed by Efron.B in 1979 [6]. There is no need to make any assumptions and restrictions on the parent distribution. After the simulated samples are sampled, the unknown parameters can be estimated through statistical prediction, which is suitable for small sample test data processing $[7,8]$. Bootstrap can be used in statistical fields such as parameter estimation, regression analysis, interval estimation, hypothesis testing and cross validation, and has been widely used in weaponry and aerospace products $[9,10]$. The Bootstrap method is not applicable to the reliability evaluation of the sample number $n=1,2$, Feng Yunwen et al [11] proposed a minimal subsample test evaluation method for the virtual augmented regenerative subsample and obtained the related reliability estimation. The Bootstrap method has also been applied to the interval estimation of parameters with a small sample on mechanical engineering field, i.e., the tank roadarm [12], the gearbox [13] and the production processing [14]. However, for the excavator working device, there is a lack of fatigue life reliability test and evaluation research.

Based on the test on site and the load spectrum of the excavator working devices, the full-scale fatigue life tests of the excavator's moving arm and the bucket rod were carried out. According to the results of the small sample fatigue test, the Bootstrap method combined with VASM was used to evaluate the actual fatigue reliability of the medium-sized excavator working devices.

\section{BOOTSTRAP METHOD}

$\mathrm{I}$ $\mathrm{t}$ is assumed that the independent random sample $\boldsymbol{X}=\left(x_{1}, x_{2}, \cdots x_{n}\right)$ comes from the unknown parent distribution $F$, and $\theta=\theta(F)$ is the unknown parameter in the parent distribution. The basic idea of the Bootstrap method is to use the sample empirical distribution function $F_{n}$ to replace the unknown parent distribution function $F$. The unknown parent parameter $\theta$ is deduced by sufficient statistics of the resampling statistics, and the steps are as follows: Step 1: For independent and identically distributed sample $\boldsymbol{X}=\left(x_{1}, x_{2}, \cdots x_{n}\right)$, the order statistics of $x_{(1)} \leq x_{(2)} \leq \cdots \leq x_{(n)}$ is obtained by sorting from small to large. The empirical cumulative distribution function $F_{n}(x)$ of samples, as demonstrated in Eqn. (1), is constructed. The estimation of $\theta$ is $\hat{\theta}=\hat{\theta}\left(F_{n}\right)$ and the estimated error is $R=\hat{\theta}\left(F_{n}\right)-\theta(F)$.

$$
F_{n}(x)=\left\{\begin{array}{cc}
0 & x<x_{(1)} \\
\frac{i}{n} & x_{(i)} \leq x \leq x_{(i+1)} \\
1 & x \geq x_{(n)}
\end{array}\right.
$$

Step 2: A sample $\boldsymbol{X}^{*}=\left(x_{1}^{*}, x_{2}^{*}, \cdots x_{n}^{*}\right)$ with a random capacity of $n$ is resampled randomly from empirical distribution function $F_{n}$, which is called Bootstrap subsample. $F_{n}^{*}$ is an empirical distribution function of $\boldsymbol{X}^{*} \cdot R^{*}=\hat{\theta}\left(F_{n}^{*}\right)-\hat{\theta}\left(F_{n}\right)$ was identified as Bootstrap statistics $R$.

Step 3: By repeating steps (2) $N$ times (usually $N \geq 1000$ ), the $N$ Bootstrap samples of $\boldsymbol{X}_{j}^{*}(j=1,2, \cdots N)$ can be obtained. The estimated $\boldsymbol{R}_{j}^{*}(j=1,2, \cdots N)$ of each Bootstrap sample statistic are calculated, and the distribution of $R$ is simulated by the distribution of $\boldsymbol{R}_{j}^{*}$, as a result, the samples $\hat{\boldsymbol{\theta}}^{*}=\left(\hat{\theta}_{1}^{*}, \hat{\theta}_{2}^{*}, \cdots, \hat{\theta}_{N}^{*}\right)$ of $\theta(F)$ are determined.

Step 4: The optimal distribution fitting, the parameter estimating and the hypothesis testing for $\hat{\boldsymbol{\theta}}^{*}$ are carried out successively, and the distribution function of the parent parameter $\theta$ is replaced by $F_{n}\left(\hat{\theta}^{*}\right)$.

Step 5: The percentile method or the corrected percentile method are commonly used to perform the interval estimation on parameter $\theta$. The Bootstrap sample estimated value of $\hat{\boldsymbol{\theta}}^{*}=\left(\hat{\theta}_{1}^{*}, \hat{\theta}_{2}^{*}, \cdots, \hat{\theta}_{N}^{*}\right)$ is arranged in ascending order. If the 
percentile method is adopted, the interval containing $(1-\alpha) \times 100 \% \hat{\boldsymbol{\theta}}^{*}$ is equal to the confidence interval of parameter $\theta$

at $(1-\alpha)$ confidence level. That is, the confidence interval of parameter $\theta$ is $\left[k_{\alpha / 2}, k_{1-\alpha / 2}\right]$, where $k_{\alpha / 2}$ and $k_{1-\alpha / 2}$ are the empirical quantiles of parameter $\theta$. Due to the shortcomings of the percentile method in the convergence of probability, the corrected percentile method is used to perform interval estimation on the unknown parameter $\theta$, then the correction of $b$ is defined as follows:

$$
b=\Phi^{-1}\left[\frac{1}{N} \sum_{j=1}^{N} I\left(\hat{\theta}_{j}^{*}\right)\right]
$$

Where $\Phi^{-1}(\bullet)$ is defined as the inverse function of standard normal distribution and $I(\bullet)$ is the indicative function, as follows:

$$
I\left(\theta_{j}\right)= \begin{cases}1, & \hat{\theta}_{j}^{*} \leq \hat{\theta} \\ 0, & \hat{\theta}_{j}^{*}>\hat{\theta}\end{cases}
$$

After correcting the parameter $\theta$, the confidence interval with confidence level $(1-\alpha)$ is $\left(k_{P L}, k_{P U}\right)$. Where $k_{P L}$ and $k_{P U}$ are the quantiles of parameter $\theta$, and $P L=\Phi\left(Z_{\alpha / 2}+2 b\right), P U=\Phi\left(Z_{1-\alpha / 2}+2 b\right)$ and $Z_{\alpha}$ are the standard normal quantiles.

\section{FATIGUE TESTING PROCEDURE AND RESULTS}

I $\mathrm{n}$ this paper, the questionnaire surveys were conducted about the material types and material proportions for medium-sized excavators (Self-weight is about $26000 \mathrm{~kg}$ ) in China. A total of 405 valid questionnaires were collected. The survey included the following excavator manufacturers: Xugong, Sunward, Sany, Hitachi, Komatsu, Doosan, Cat, Hyundai and Liugong. According to the statistical analysis on the survey data and the material conditions of the load spectrum test site, the type and proportion of the materials for the medium-sized excavator were determined with reference to Standard for engineering classification of soil [15], which were as follows: loose soil (24.6\%), sub clay $(22.6 \%)$, clay $(24.1 \%)$, and heavy clay containing clay $(28.7 \%)$. Finally, the load spectrum tests of excavators were completed on the test site, and the load spectrums of the excavator working devices were obtained.

Due to the changing of the attitude of the excavator working devices (moving arm and bucket rod) in the actual operation, the fatigue loading schemes on the platform under the local coordinates of the moving arm and the bucket rod were put forward. For the complex loads on moving arm and bucket rod, a load equivalent method (LEM) based on the maximum bending moment cross section was proposed. In order to verify equivalent loads, the stress histories of major stress points on moving arm and bucket rod were calculated by finite element analysis, and the stress histories were compared with the measured stress histories obtained by testing onsite. The location of the measuring points is shown in Fig. 2, and the comparison results are shown in Fig. 3. Using the Corrcoef function in Matlab software, it is concluded that the correlation between the measured points and the measured stress histories under the 95\% confidence level is $88.6 \% \sim 97.21 \%$. The damage equivalent criterion [16] was used to correct the equivalent vertical load of moving arm and bucket rod, and the fatigue test load spectrums of moving arm and bucket rod on medium-sized excavators were obtained. The schematic diagrams of fatigue test are shown in Fig. $1 . F_{e q}$ and $F_{k e q}$ are fatigue test loads for moving arm and bucket rod respectively. For details, see the previous paper [17].

The full-scale fatigue tests of 2 sets of excavators' moving arm and bucket rod were completed, as shown in Fig. 2. In order to ensure uniformity of the manufacturing quality of the moving arm and bucket rod, the robotized welding is mainly used. During the experiment, the loading frequency is $1-2 \mathrm{~Hz}$, and the penetration crack or crack propagation to about $5 \mathrm{~cm}$ is the criterion of stopping. The fatigue test results are illustrated in Tab. 1 and the fatigue failures are shown in Fig. 4 and Fig. 5. In addition, according to the experience of the industry, the life target of the excavator working device is about $8000 \sim 10000$ hours. Thus, during the fatigue test, the testing machine will be stopped when the fatigue life is over 
11000 hours for the members without fatigue crack. The shorter fatigue life of the moving arm and bucket rod is identified as the fatigue life of the excavator. Therefore, the fatigue life of the 2 sets of excavators is $T_{1}=9870 \mathrm{~h}$ and $T_{2}=4179 \mathrm{~h}$ respectively.

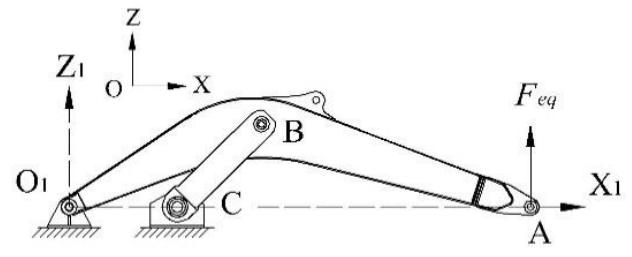

(a) Moving arm of excavator

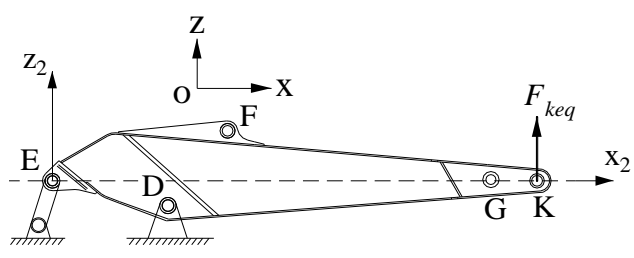

(b) Bucket rod of excavator

Figure 1: Schematic diagrams of the loading scheme during fatigue testing. and are the fatigue test loads of moving arm and bucket rod on platform frame respectively, and $\mathrm{O} 1, \mathrm{~B}, \mathrm{E}$ and $\mathrm{D}$ are hinged points. The link of BC is used to support moving arm.

\begin{tabular}{|c|c|c|c|c|c|}
\hline \multirow{2}{*}{ No. } & \multicolumn{2}{|r|}{ Moving arm } & \multicolumn{2}{|r|}{ Bucket rod } & \multirow{2}{*}{$\begin{array}{c}\text { Fatigue life for } \\
\text { working devices } \\
\text { of excavator } \\
/ \mathrm{h}\end{array}$} \\
\hline & $\begin{array}{c}\text { Fatigue life } \\
/ \mathrm{h}\end{array}$ & Failure location & $\begin{array}{l}\text { Fatigue life } \\
/ \mathrm{h}\end{array}$ & Failure location & \\
\hline 1 & 10470 & $\begin{array}{l}\text { The front end of } \\
\text { welding toe at the } \\
\text { supporting plate on } \\
\text { the upper flange plate } \\
\text { of the moving arm, as } \\
\text { shown in Fig. 4(a). }\end{array}$ & 9870 & $\begin{array}{l}\text { The front end of welding } \\
\text { toe at the supporting plate } \\
\text { on the upper flange plate } \\
\text { of the bucket rod, as } \\
\text { shown Fig. 4(b). }\end{array}$ & 9870 \\
\hline 2 & 4179 & $\begin{array}{l}\text { The front end of } \\
\text { welding toe at the } \\
\text { supporting plate on } \\
\text { the upper flange plate } \\
\text { of the moving arm, as } \\
\text { shown in Fig. } 5 \text {. }\end{array}$ & $>11000$ & No failure & 4179 \\
\hline $\begin{array}{l}\text { Mean } \\
\text { value }\end{array}$ & & & & & 7024.5 \\
\hline
\end{tabular}

Table 1: Fatigue test results of excavator working devices.

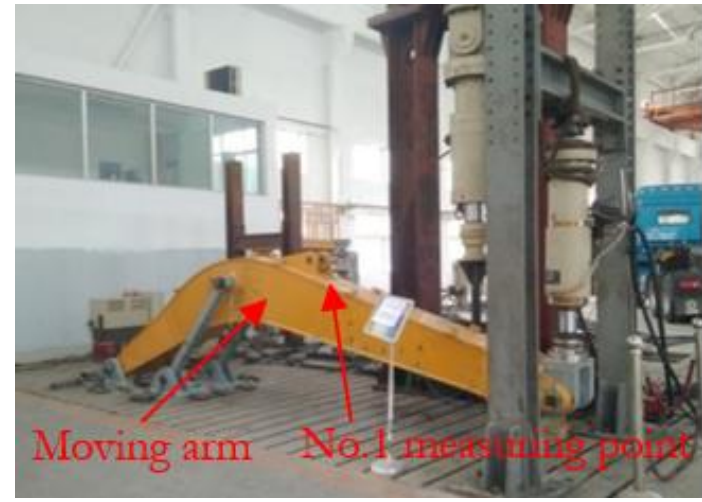

(a) Moving arm

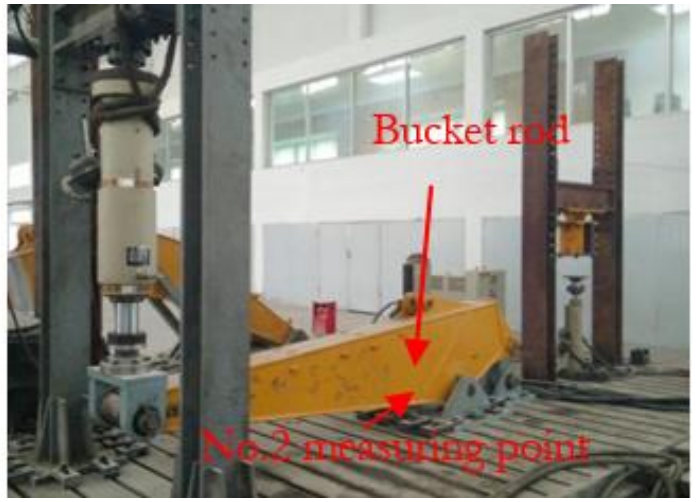

(b) Bucket rod

Figure 2: Fatigue test photo of working device of medium-sized excavator. 


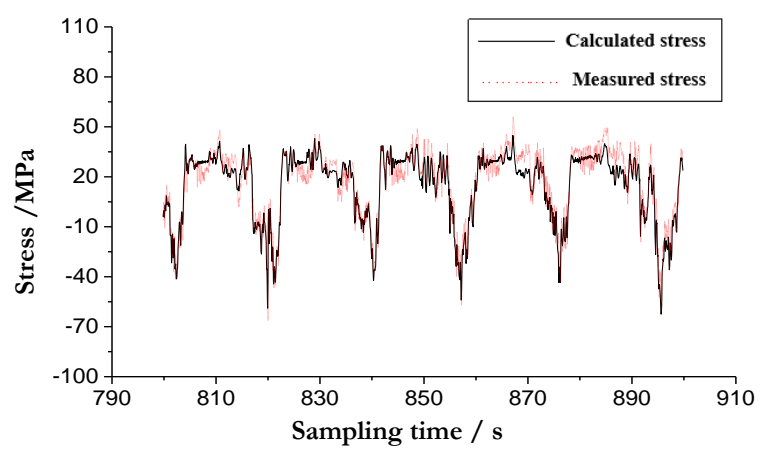

(a) No.1 measuring point on moving arm

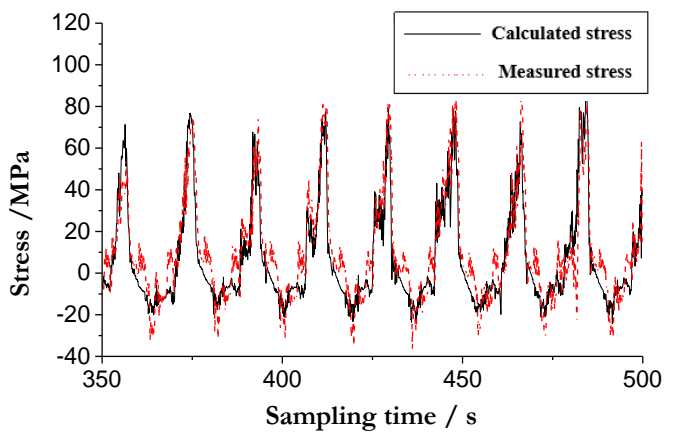

(b) No.2 measuring point on bucket rod

Figure 3: Comparisons of calculated stress and measured stress at measuring points

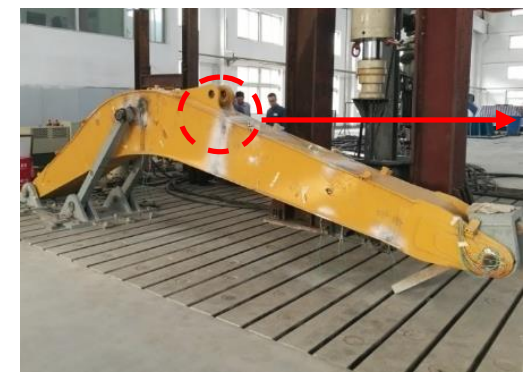

(i)

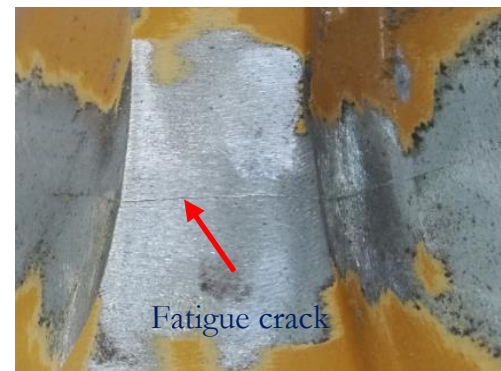

(ii) Enlarged view

(a) Fatigue failures on moving arm

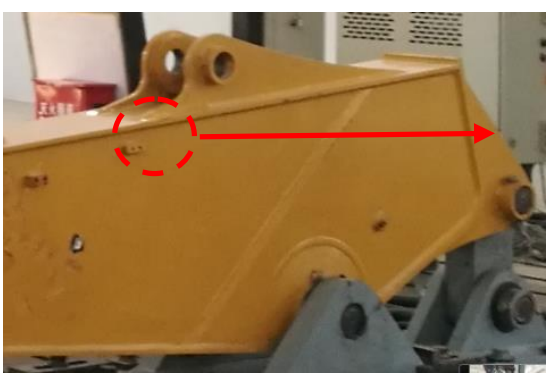

(i)

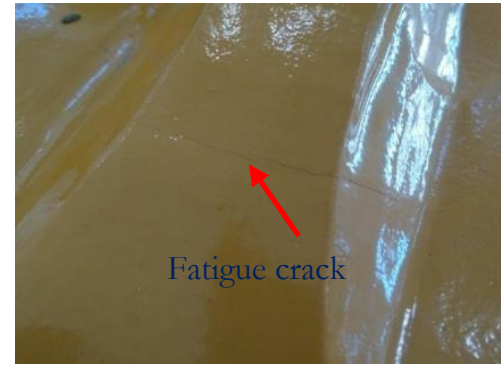

(ii) Enlarged view

(b) Fatigue failures on bucket rod

Figure 4: Fatigue failures on No.1 excavator

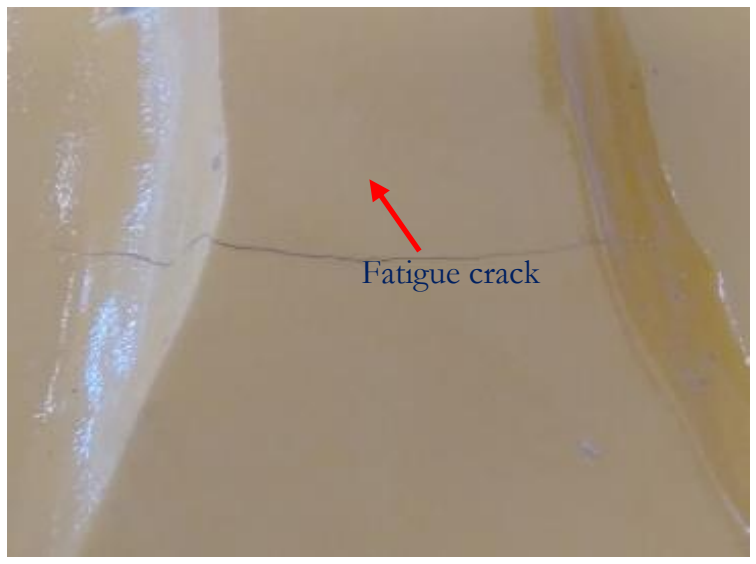

Figure 5: Fatigue failures on moving arm of No.2 excavator, which is the same as failure position of No.1 excavator 


\section{RELIABILITY EVALUATION ON SMALL-SIZE SAMPLE OF EXCAVATOR WORKING DEVICES}

he statistical distribution of fatigue life of structural parts is generally considered to obey lognormal distribution or Weibull distribution. In this study, the fatigue life of the excavator working device is assumed to be logarithmic normal distribution, and then the logarithmic fatigue life $Y=\lg T$ obeys the normal distribution. Its mean value is $\mu_{Y}$, and the standard deviation is $\sigma_{Y}$. Under certain reliability $R$, the logarithmic safety life $y_{R}$ of the excavator working device is defined as:

$$
P\left(Y>y_{\mathrm{R}}\right)=\int_{y_{\mathrm{R}}}^{\infty} f(y) d y=R
$$

Where $f(y)$ is the probability density function of the logarithmic life. The logarithmic safety life $y_{R}=\mu_{Y}+u_{R} \sigma_{Y}$ can be obtained according to Eqn. (4), and its estimated value is as follows:

$$
\hat{y}_{R}=\hat{\mu}_{Y}+u_{R} \hat{\sigma}_{Y}
$$

where:

$\hat{\mu}_{Y}$ is the mean value of the parent;

$\hat{\sigma}_{Y}$ is the estimated standard deviation of the parent;

$u_{R}$ is a standard normal deviation corresponding to reliability $R$, that is $u_{R}=\Phi^{-1}(1-R)$.

The Bootstrap method is usually applied to small sample test evaluation with sample size of $n>10$. As the test sample of the excavator working device was only 2 sets in this study, the VASM was used to augment the original sample $n=2$ to the sample $n^{*}=12$. The basic principle of the VASM is that the average value and the standard deviation of the augmented sample should be equal to those of the original test. The recommended virtual augmented empirical formula is [11]:

$$
y_{i, i+m / 2}=\bar{y} \pm\left[0.017 \times(i-1)^{3}+\xi\right] \sigma, \quad i=1,2, \cdots m / 2
$$

where:

$y$ is an augmented sample value;

$\bar{y}$ is the mean life mean of the test sample;

$\sigma$ is a standard deviation of the subsample of a similar part;

$m$ is an augmented sample number;

$\xi$ is the undetermined coefficient.

An augmented sample obtained by the Eqn. (6) was:

$$
\begin{aligned}
& y_{1}=\bar{y}-\xi \sigma, y_{2}=\bar{y}-(0.017+\xi) \sigma, \\
& y_{3}=\bar{y}-\left(0.017 \times 2^{3}+\xi\right) \sigma, y_{4}=\bar{y}-\left(0.017 \times 3^{3}+\xi\right) \sigma, \\
& y_{5}=\bar{y}-\left(0.017 \times 4^{3}+\xi\right) \sigma, y_{6}=\bar{y}+\xi \sigma, \\
& y_{7}=\bar{y}+(0.017+\xi) \sigma, y_{8}=\bar{y}+\left(0.017 \times 2^{3}+\xi\right) \sigma, \\
& y_{9}=\bar{y}+\left(0.017 \times 3^{3}+\xi\right) \sigma, y_{10}=\bar{y}+\left(0.017 \times 4^{3}+\xi\right) \sigma, \\
& y_{11}=\lg 4197, y_{12}=\lg 9870
\end{aligned}
$$

From the fatigue test results of the excavator working device, the mean value of logarithmic fatigue life $\bar{y}=\frac{1}{n} \sum_{i=1}^{n} \lg T_{i}=3.8077$ and standard deviation $\sigma_{y}=0.2639$ of the sample were achieved. According to the basic principle of VASM, the equations were as follows: 


$$
\left\{\begin{array}{c}
\frac{1}{12} \sum_{j=0}^{12} y_{j}=\bar{y} \\
\frac{1}{11} \sum_{j=0}^{12}\left(y_{j}-\bar{y}\right)^{2}=\sigma_{y}{ }^{2}
\end{array}\right.
$$

The augmented sample $y_{1} \sim y_{12}$ was substituted to the Eqn. (7), and $\xi=0.5727$ was obtained. The augmented samples arranged in order were $Y=\{3.3694,3.5354,3.6207,3.6211,3.6521,3.6566,3.9588,3.9633,3.9943,3.9947$, $4.0800,4.2460\}$.

The empirical cumulative distribution function $F_{n}(y)$ of the augmented sample was set up, as shown in Fig. 6. MonteCarlo method was used to resample the $N$ groups of Bootstrap samples from the empirical cumulative distribution function $F_{n}(y)$, that is $y^{(k)}=\left(y_{1}^{(k)}, y_{2}^{(k)}, \cdots y_{n^{*}}^{(k)}\right),(k=1,2, \cdots N)$.

The mean value of Bootstrap sample mean value and the mean value of Bootstrap sample standard deviation with sampling number were illustrated in Fig. 7. The results showed that when the number of Bootstrap samples was $N \geq 10000$, the mean values of Bootstrap sample mean value and Bootstrap sample standard deviation reached a stable state. Therefore, the number of Bootstrap sampling number was $N=10000$. The mean value and standard deviation of 10000 sets of Bootstrap samples were statistically analyzed, and the distribution characteristics of logarithmic life mean $\mu_{Y}$ and standard deviation $\sigma_{Y}$ were estimated. K-S test results showed that the logarithmic life mean and standard deviation followed normal distribution, and $\mu_{Y} \sim N\left(3.8120,0.0645^{2}\right), \sigma_{Y} \sim N\left(0.2221,0.0305^{2}\right)$. The frequency distribution histograms and normal distribution fitting curves of Bootstrap sample mean value and its standard deviation were shown in Fig. 8 and 9 respectively.

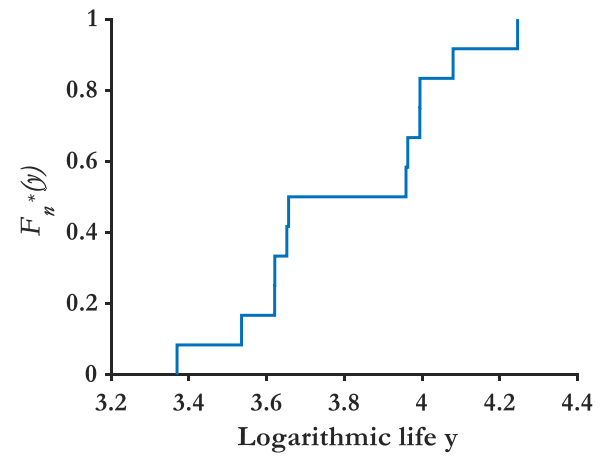

Figure 6: Empirical distribution function of augmented logarithmic life sample $Y$.

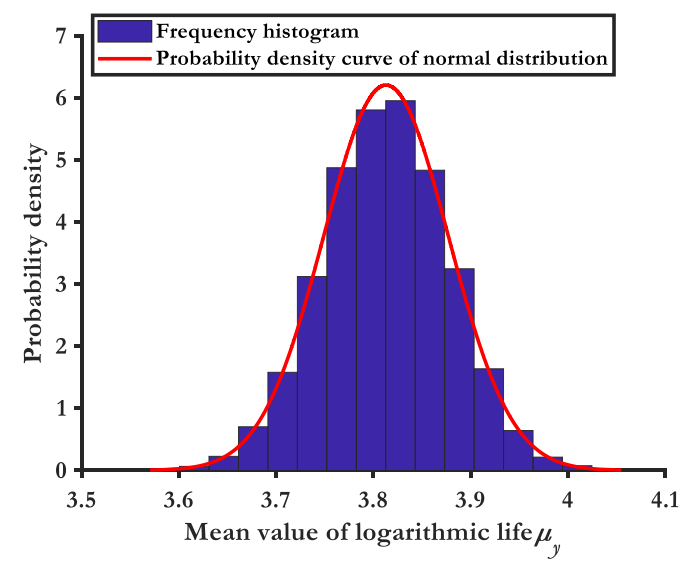

Figure 8: The frequency distribution histogram and curve fitting of Bootstrap sample mean value

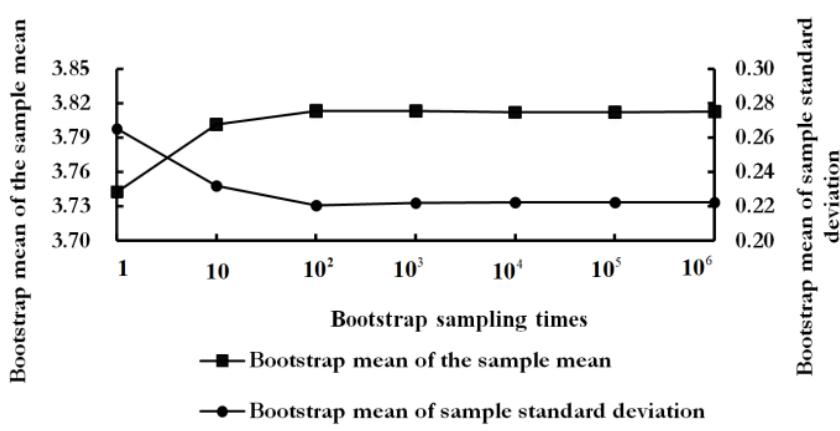

Figure 7: Logarithmic life parameter estimation varies with Bootstrap simulations.

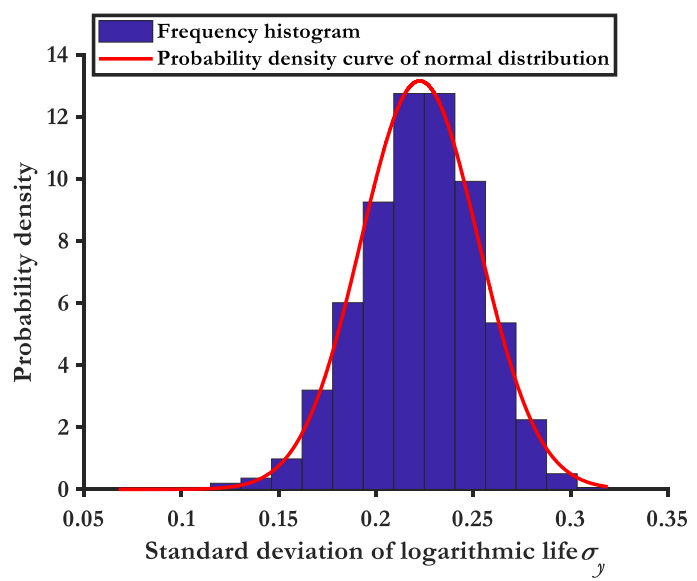

Figure 9: The frequency distribution histogram and curve fitting of Bootstrap sample standard deviation 
Referring to the international fatigue strength design standard for welded structures [18], the general reliability is $75 \%$. Therefore, the confidence interval of the $75 \%$ confidence level of the mean logarithmic life was estimated to be [3.7311,3.8793] using the Bootstrap corrected percentile method. According to the student-t distribution theory, the confidence interval of the logarithmic life expectancy at the $75 \%$ confidence level was $[3.3571,4.2582]$. By comparing the two results, the average confidence interval obtained by Bootstrap method was narrow, and the accuracy of interval estimation was improved.

In practice, the confidence lower limit of the percentile value of the parent is often used to estimate the true value of logarithmic safety life. Thus, the lower confidence limit of percentile value of the logarithmic life expectancy at the $75 \%$ confidence level was $\mu_{Y}=3.7311$. Estimated value of Bootstrap sample standard deviation $\sigma_{Y}=0.2221$ was adopted as the standard deviation of the parent logarithmic life. The above estimated values were substituted in the Eqn. (5), and the reliability indexes of the working device of the excavator were calculated as follows:

Reliably function $R(t)$ is

$$
R(t)=1-\Phi\left(\frac{\lg t-3.7311}{0.2221}\right)
$$

The reliability function curve of excavator working device under $75 \%$ confidence level was shown in Fig. 10. As the working hours increasing, the reliability of working devices will be decreased. When the working time reaches $0 \sim 2300$ hours, the reliability is gradually reduced. The working time is $2300 \sim 7000$ hours, and the reliability is rapidly reduced. But when the working time is 7000 hours, the reliability decreases slowly. The working hours is closing to 15000 hours, and the reliability tends to 0 .

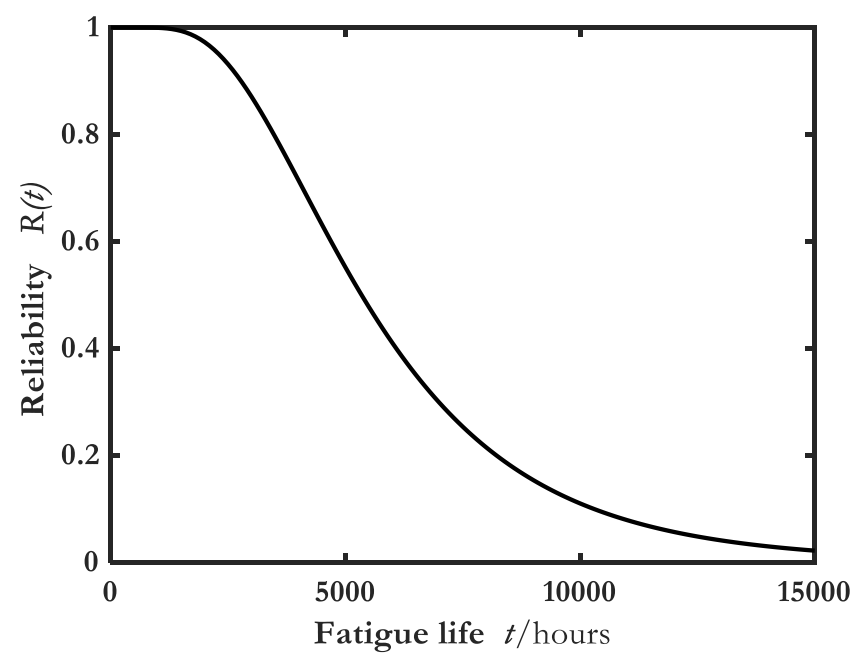

Figure 10: Reliability function curve of excavator working device

(2) The failure distribution function $F(t)$ and the probability density function $f(t)$ are

$$
\begin{aligned}
& F(t)=1-R(t)=\Phi\left(\frac{\lg t-3.7311}{0.2221}\right) \\
& f(t)=\frac{1}{\sigma_{Y} t \sqrt{2 \pi} \ln 10} \exp \left[-\frac{1}{2}\left(\frac{\lg t-\mu_{Y}}{\sigma_{Y}}\right)^{2}\right]=\frac{1}{0.2221 \sqrt{2 \pi} t \ln 10} \exp \left[-\frac{1}{2}\left(\frac{\lg t-3.7311}{0.2221}\right)^{2}\right]
\end{aligned}
$$

The failure probability density function curve of the excavator working device was shown in Fig. 11. It can be seen that the failure probability is $f(t)_{\max }=1.6586 \times 10^{-4}$ when $t \approx 4400 \mathrm{~h}$. This indicates that when the working device test sample runs to $4400 \mathrm{~h}$, the failure samples account for about $0.0166 \%$ of the total sample size. 


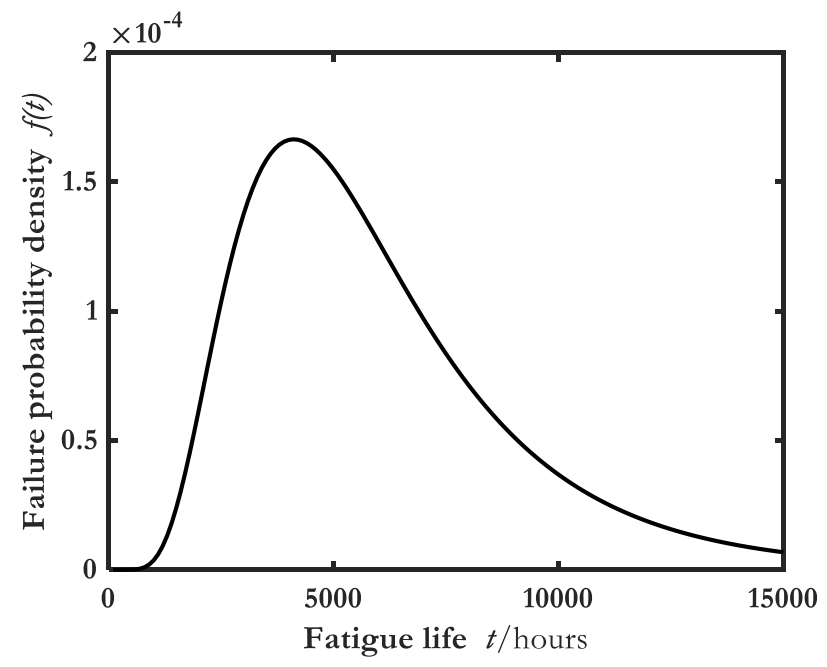

Figure 11: Failure probability function curve of excavator working device

(3) Inefficiency function is

$$
\lambda(t)=\frac{f(t)}{R(t)}=\frac{\phi\left(\frac{\lg t-3.7311}{0.2221}\right) / t \ln 10}{1-\Phi\left(\frac{\lg t-3.7311}{0.2221}\right)}
$$

The failure rate function curve of the excavator working device was shown in Fig. 12. Without preventive maintenance, the failure rate function of the excavator working device is incremental, and the increasing speed is faster, then slows down, and finally tends to be stable. When the working time is $0 \sim 1800$ hours, the failure probability is increased slowly. This stage is an occasional failure stage. At this stage, we should do a good job in maintenance of the excavator work device, so that the working time of the product can be prolonged as long as possible. When the working time is $1800 \sim 4800$ hours, the failure probability is increased rapidly. This stage is mainly caused by the fatigue damage and abrasion of the excavator working device. If the preventive maintenance or updating parts are to be applied at this time, the failure rate can be reduced to ensure the reliable operation of the excavator working device.

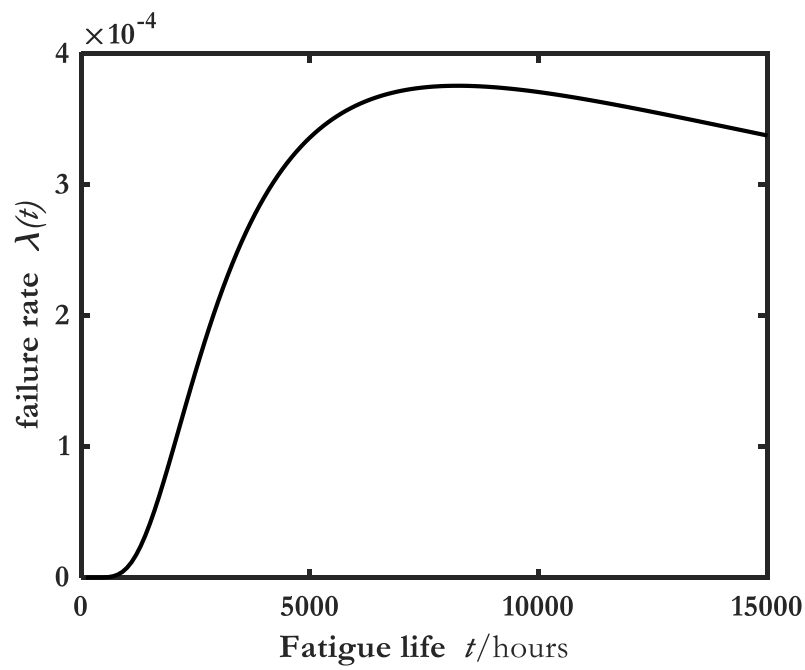

Figure 12: Failure rate curve of excavator working device 
(4) Reliablelife is

$$
t_{\mathrm{R}}=10^{\left(\mu_{Y}-\sigma_{Y} \beta\right)}=10^{\left[3.7311+0.2221 \Phi^{-1}(1-R)\right]}
$$

By using the above method, the fatigue life evaluation results of the excavator working device under different confidences and reliabilities are calculated, as shown in Tab. 2.

\begin{tabular}{|c|c|c|c|c|c|c|c|}
\hline \multirow{2}{*}{ Confidence level } & \multicolumn{6}{|c|}{ Reliability } & \multirow{2}{*}{$\begin{array}{c}\text { Average } \\
\text { fatigue } \\
\text { life }\end{array}$} \\
\hline & $36.8 \%$ & $50 \%$ & $75 \%$ & $90 \%$ & $95 \%$ & $99 \%$ & \\
\hline $75 \%$ & 6384 & 5372 & 3803 & 2787 & 2314 & 1633 & 6124 \\
\hline $90 \%$ & 5909 & 4974 & 3525 & 2585 & 2147 & 1516 & 5667 \\
\hline $95 \%$ & 5647 & 4753 & 3367 & 2469 & 2051 & 1448 & 5416 \\
\hline
\end{tabular}

Table 2: Fatigue life evaluation results of excavator working device (h)

\section{CONCLUSIONS}

(1) According to the load characteristics of the excavator working devices, i.e., the moving arm and the bucket rod, the equivalent load models under fixed attitude were established based on the load equivalent method (LEM). The schemes for fatigue test of excavator working devices in a local coordinate system were designed to overcome the contradiction between the variable posture during working and the fixed attitude during fatigue testing. The full-scale fatigue life tests of 2 sets of medium-sized excavators' moving arm and bucket rod were carried out respectively.

(2) According to the data of the minimum sample fatigue life test of the excavator working device, the reliability evaluation of the excavator working device was carried out by the Bootstrap method combined with virtual augmented sample method (VASM). By this way, the reliability function, the failure distribution function, the failure rate function and the reliable life were obtained. The evaluation results show that the reliability of the excavator working device varying with time is basically consistent with the performance degradation rule of mechanical products. The analysis results can provide a reference for the detection and maintenance of the excavator working devices.

(3) The fatigue lives of excavator working device under different confidence and reliability were obtained. Under the $75 \%$ confidence level, the average working time of the excavator working device is 6124 hours, which is far from the expected 8000-10000 hours in the line. Fatigue design method and fatigue tests should be adopted to optimize the structures and gradually improve the fatigue lives of excavator structural parts.

\section{ACKNOWLEDGEMENTS}

his work is supported by the National Science-technology Support Program Project (No. 2015BAF07B02), the Shanxi National Science Foundation project (No. 2017JM5040) and the Fundamental Research Funds for the Central Universities (No. 300102258508).

\section{REFERENCES}

[1] Illera, D., Pugliese, V., Mesa, J., et al. (2018). Failure assessment of a weld-cracked mining excavator boom, Eng. Failure Anal., 90(8), pp. 47-63. DOI: 10.1016/j.engfailanal.2018.03.022

[2] Zheng, G., Yang, X., Zhou, H., et al. (2018). A simplified prediction method for evaluating tunnel displacement 
induced by laterally adjacent excavations, Comput. Geotech., 95, pp. 119-128. DOI: 10.1016/j.compgeo.2017.10.006

[3] Gran, B. A., and Helminen, A. (2001). A Bayesian Belief Network for Reliability Assessment, Computer Safety, Reliability and Security, Springer Berlin Heidelberg, pp. 35-45.

[4] Ahsanullah, M., Kibria, B. M. G., Shakil, M. (2014). Characterizations of Student's t Distribution. Atlantis Press, pp. 129-141.

[5] Saberi, M. R., Rahai, A. R., Sanayei, M., et al. (2017). Steel Bridge Service Life Prediction Using Bootstrap Method, Int. J. Civ. Struct. Eng., 15(1), pp.1-11. DOI: 10.1007/s40999-016-0036-z

[6] Efron, B. (1992). Bootstrap Methods: Another Look at the Jackknife, Breakthroughs in Statistics. Springer New York, pp. $1-26$.

[7] Seruga, D., Nagode, M. (2015). A method for long-term creep-rupture strength prediction based on a small sample of experimental results, smoothed bootstrapping and time-temperature parameters, Mater. Des., 67, pp. 180-187. DOI: 10.1016/j.matdes.2014.11.011

[8] Wang, Y., Zhou, W., Dong, D., et al. (2017). Estimation of random vibration signals with small samples using bootstrap maximum entropy method, Meas, 105, pp. 45-55. DOI: 10.1016/j.measurement.2017.04.003

[9] Peng, W., Fang, Y. W., Zhan, R., et al. (2016). Weapon systems accuracy evaluation using the error spectrum, Aerosp. Sci. Technol., 58, pp. 369-379. DOI: 10.1016/j.ast.2016.08.032

[10] Muller, L., Roche, J. M., Hurmane, A., et al. (2018). Experimental monitoring of the self-heating properties of thermoplastic composite materials, Procedia Eng., 213, pp. 183-191. DOI: 10.1051/matecconf/201816507003

[11] Hung, W., Feng, Y., Lu, Z. (2005). Virtually expanded sample estimation method for extremely small-scale sample test, J. Northwest. Polytech. Univ., 23(3), pp. 384-387. DOI: 10.3969/j.issn.1000-2758.2005.03.024 (In Chinese)

[12] Noh, Y., Choi, K. K., Lee, I., et al. (2010). Reliability-Based Design Optimization with Confidence Level for NonGaussian Distributions Using Bootstrap Method, J. Mech. Des., 133(9), pp. 1065-1076. DOI: 10.1115/1.4004545

[13] Wang, H., Deng, X., Li, J., et al. (2015). Dynamic Assessment of Vibration of Tooth Modification Gearbox Using Grey Bootstrap Method, Shock Vib., 4, pp. 1-8. DOI: 10.1155/2015/204609

[14] Niaki, S. T., Abbasi, B. (2007). Bootstrap method approach in designing multi-attribute control charts, Int. J. Adv. Manuf. Technol., 35(56), pp. 434-442. DOI: 10.1007/s00170-006-0728-7

[15] "Standard for engineering classification of soil", GB/T 50145-2007, National Standards of the People's Republic of China, pp. 8-10. (In Chinese)

[16] Zhang, S. G. (2008). Research on load spectrum test and establishment method of high speed train bogie, Sci. China, 38(11), pp. 1805-1814. DOI: 10.3321/j.issn:1006-9275.2008.11.002 (In Chinese)

[17] Xiang, Q. Y., Lu, P. M., Wang, B. H., et al. (2017). Load spectrum test method of hydraulic excavator working device, China J. Highw. Transp., 30(9), pp. 151-158. DOI: 10.3969/j.issn.1001-7372.2017.09.019 (In Chinese)

[18] Hobbacher, A., F. (2016). Recommendations for Fatigue Design of Welded Joints and Components. Springer International Publishing. 